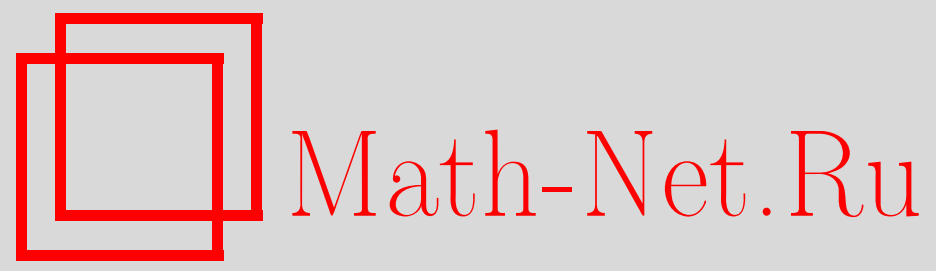

Обцероссийский математический портал 
В. А. Демьяненко, К гипотезе Мазура, Матем. заметки, 1998, том 63, выпуск 2, 294-296

DOI: https://doi.org/10.4213/mzm1277

Использование Общероссийского математического портала Math-Net.Ru подразумевает, что вы прочитали и согласны с пользовательским соглашением 
http://www. mathnet.ru/rus/agreement

Параметры загрузки:

IP : 54.147 .182 .235

26 апреля 2023 г., 09:36:45 


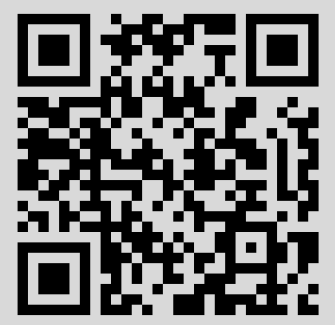




\section{К ГИПОТЕЗЕ МАЗУРА}

\section{В. А. Демьяненко}

Пусть $n$ - произвольное натуральное число, $\varepsilon(n)=e^{2 \pi i / n}, \mathbb{Q}$ - поле рациональных чисел, $\mathbb{Q}(\varepsilon(n))$ - поле, полученное присоединением к $\mathbb{Q}$ единицы $\varepsilon(n), F: y^{2}=x^{3}+r x+s$ - эллиптическая кривая, определенная над $\mathbb{Q}(\varepsilon(n)), D=-4 r^{3}-27 s^{2} \neq 0,\left\{O_{m}(F), O_{m}^{\prime}(F)\right\}$ - базис всех точек порядка $m$ на $F$ и $\alpha O_{m}(F)+\beta O_{m}^{\prime}(F)=\left\{x_{\alpha / m, \beta / m}, y_{\alpha / m, \beta / m}\right\}$.

Цель настоящей заметки - доказательство двух следующих теорем.

Теорема 1. Если $O_{2^{\alpha}}(F), 2^{\alpha-1} O_{2^{\alpha}}^{\prime}(F)=O_{2}^{\prime}(F) \in \mathbb{Q}\left(\varepsilon\left(2^{t}\right)\right)(t$ произвольное $)$, то $\alpha \leqslant 3$.

Теорема 2. Если $O_{3} \beta(F), O_{3}^{\prime}(F) \in \mathbb{Q}\left(\varepsilon\left(3^{t}\right)\right)$ ( $t$ произвольное $)$, то $\beta \leqslant 1$. 
ДоКАЗАТЕЛЬСТВО ТЕОРЕМЫ 1 . Пусть $\mathbb{k}$-алгебраическое поле, эллиптическая кривая $F$ определена над $\mathbb{k}, P$ - произвольная $\mathbb{k}$-точка на $F$ и $t P=\left\{x_{t}, y_{t}\right\}$. Так как

$$
2 y_{1} \sqrt{x_{2}-\rho}=\left(x_{1}-\rho\right)^{2}-\left(r+3 \rho^{2}\right), \quad \rho^{3}+r \rho+s=0,
$$

в силу (1) кривая $G: v^{2}=u^{4}+a u^{2}+b, a=3 \rho, b=r+3 \rho^{2}$, имеет точки $t Q=\left\{u_{t}, v_{t}\right\}$, $u_{t}=\sqrt{x_{2 t}-\rho}, v_{t}=y_{2 t} / \sqrt{x_{2 t}-\rho}$, для которых, как и в $(1)$, имеем

$$
\pm u_{2 t}=\frac{u_{t}^{4}-b}{2 u_{t} v_{t}}, \quad \pm v_{2 t}=\frac{u_{t}^{8}+2 a u_{t}^{6}+6 b u_{t}^{4}+2 a b u_{t}^{2}+b^{2}}{4 u_{t}^{2} v_{t}^{2}}
$$

Из (2) получаем

$$
\left(\frac{v_{t}}{u_{t}}\right)^{2}= \pm 2 v_{2 t}+a+2 u_{2 t}^{2}, \quad u_{t}^{2}=u_{2 t}^{2} \pm v_{2 t} \pm u_{2 t}\left(\frac{v_{t}}{u_{t}}\right) .
$$

Полагая в (3) $t=2,4, \ldots$ и учитывая (1), выводим

$$
\begin{gathered}
O_{16}(F)=\left\{x_{1 / 16,0}, y_{1 / 16,0}\right\}, \quad O_{2}^{\prime}(F)=\left\{x_{0,1 / 2}, y_{0,1 / 2}\right\} \in \mathbb{k}, \\
Y^{2}=X^{3}+4 X, \quad X, Y \in \mathbb{k}, X=2 T^{2}, \quad Y=2 T\left(\alpha\left(T^{2}-1\right)-T^{2}-1\right), \\
T=\frac{\left(\rho_{2}-\rho_{3}\right)\left(1-\alpha^{2}\right)^{2}}{\left(1+\alpha^{2}\right)\left(\alpha^{2}-2 \alpha-1\right)}, \quad \alpha=\frac{2 u_{1 / 16,0}}{\left(v_{1 / 16,0} / u_{1 / 16,0}+1\right)^{2}}, \\
u_{1 / 16,0}=\sqrt{x_{1 / 8,0}-\rho_{1}}=\frac{\left(x_{1 / 16,0}-\rho_{1}\right)^{2}-r-3 \rho_{1}^{2}}{2 y_{1 / 16,0}}, \quad \frac{v_{1 / 16,0}}{u_{1 / 16,0}}=\frac{y_{1 / 8,0}}{x_{1 / 8,0}-\rho_{1}}, \\
\left\{x_{1 / 2,0}, y_{1 / 2,0}\right\}=\left\{\rho_{1}, 0\right\}, \quad\left\{x_{0,1 / 2}, y_{0,1 / 2}\right\}=\left\{\rho_{2}, 0\right\}, \quad\left\{x_{1 / 2,1 / 2}, y_{1 / 2,1 / 2}\right\}=\left\{\rho_{3}, 0\right\}, \\
\rho_{i}^{3}+r \rho_{i}+s=0, \quad i=1,2,3 .
\end{gathered}
$$

Теперь если $\mathbb{k}=\mathbb{Q}\left(\varepsilon\left(2^{t}\right)\right)\left(t\right.$ произвольное) и $O_{16}(F) \in \mathbb{Q}\left(\varepsilon\left(2^{t}\right)\right)$, то (4) противоречит результатам работы [1]. Следовательно, $\alpha \leqslant 3$. Теорема 1 доказана.

ДоКАЗАТЕЛЬСТво ТЕОРЕмЫ 2. Предположим, что над произвольным алгебраическим полем $\mathbb{k}$ $O_{9}(F), 3 O_{9}^{\prime}(F)=O_{3}^{\prime}(F) \in \mathbb{k}$. Согласно [2]

$$
\begin{gathered}
u^{3}-v^{3}=1, \quad u, v \in \mathbb{k}, \quad u_{j}=\frac{1}{2} \sqrt[3]{\frac{3 r+9 x_{\left(c_{j} / 3\right)}^{2}}{x_{\left(c_{j} / 3\right)}^{2}}}, \quad v_{j}=\frac{1}{2} \sqrt[3]{\frac{3 r+x_{\left(c_{j} / 3\right)}^{2}}{x_{\left(c_{j} / 3\right)}^{2}},} \\
c_{1, j} O_{3}+c_{2, j} O_{3}^{\prime}=\left\{x_{\left.\left(c_{j} / 3\right), y_{\left(c_{j} / 3\right)}\right\}}, \quad\left(c_{j} / 3\right)=\{1 / 3,0\},\{0,1 / 3\},\{1 / 3,1 / 3\},\{1 / 3,-1 / 3\},\right. \\
\sqrt[3]{\frac{3 r+x_{1 / 3,0}^{2}}{x_{1 / 3,0}^{2}}}=\frac{1}{2} x_{1 / 3,0}^{-1} \sum_{t=0}^{2} \varepsilon^{-t}(3) x_{t / 3,1 / 3}, \\
\sqrt[3]{\frac{3 r+9 x_{1 / 3,0}^{2}}{x_{1 / 3,0}^{2}}}=\frac{1}{6} x_{1 / 3,0}^{-1} \sum_{\alpha, \beta=0}^{2} \varepsilon^{-\beta}(3) x_{(3 \alpha+1) / 9, \beta / 3},
\end{gathered}
$$

где в силу [3]

$$
\frac{y_{1 / 3,1 / 3}}{x_{1 / 3,1 / 3}-x_{0,1 / 3}} / \frac{y_{1 / 3,0}}{x_{1 / 3,0}-x_{0,1 / 3}}=\varepsilon(3)=\frac{-1-\sqrt{-3}}{2} .
$$

Теперь если $\mathbb{k}=\mathbb{Q}\left(\varepsilon\left(3^{t}\right)\right)$ ( $t$ произвольное), то (5) и (6) противоречат результатам работы [4]. Следовательно, $\beta \leqslant 1$. Теорема 2 доказана.

Пусть $\mathbb{k}$ - алгебраическое поле, $F_{1}$ и $F_{2}$ - эллиптические кривые, определенные соответственно над полями $\mathbb{k}$ и $\mathbb{k}\left(\varepsilon\left(m^{t}\right)\right)\left(t\right.$ произвольное) и $\Gamma_{1}(m), \Gamma_{2}(m)$ - группы точек $m$-кручения $F_{1}$ и $F_{2}$.

Доказанные теоремы позволяют выдвинуть следующие гипотезы. 
ГипотезА 1. Если $O_{m}\left(F_{1}\right), O_{m}^{\prime}\left(F_{1}\right) \in \mathbb{k} u O_{m}\left(F_{2}\right), O_{m}^{\prime}\left(F_{2}\right) \in \mathbb{k}\left(\varepsilon\left(m^{t}\right)\right), m o \Gamma_{1}(m) \cong \Gamma_{2}(m)$.

ГИпотезА 2. Если для некоторой эллиптической кривой $F_{2}$ и натурального числа $m$ $O_{m}\left(F_{2}\right) \in \mathbb{k}\left(\varepsilon\left(m^{t}\right)\right)$, то для некоторой кривой $F_{1}$ и того же числа $m \quad O_{m}\left(F_{1}\right) \in \mathbb{k}$.

\section{СПИСОК ЦИТИРОВАННОЙ ЛИТЕРАТУРЫ}

1. Башмаков М. И., Кириллов А. С. // Записки научн. семин. ЛОМИ. 1976. Т. 57. С. 5-7. 2. Демьяненко В. А. // Матем. заметки. 1985. Т. 37. № 1. С. 99-102. 3. Демьяненко В. А. // Записки научн. семин. ЛОМИ. 1983. Т. 111. С. 58-61. 4. Башмаков М. И., аль-Надер. // Матем. сб. 1973. Т. 90. № 1. С. 117-130.

Институт математики и механики УрО РАН 